\title{
A Comparative Study on Methods of Distinction Between Near- and Far-Side Defects as Techniques Used Alongside with the Magnetic Flux Leakage Testing
}

\author{
Zbigniew Usarek $^{1,2}$ (1) Marek Chmielewski ${ }^{1,2} \cdot$ Leszek Piotrowski $^{1,2}$
}

Received: 17 March 2021 / Accepted: 9 January 2022 / Published online: 22 January 2022

(c) The Author(s) 2022

\begin{abstract}
Results of the finite element analysis show that a far-side defect in a steel plate, with the depth greater by $10 \%$ of the plate thickness than a near-side defect, can produce a very similar magnetic flux leakage (MFL) signal. Due to the fact that a measurement of MFL itself can lead to misclassification of a far-side defect as a near-side one, and thus to underestimation of its depth, a comparative study of three complementary magnetic techniques was performed. The following techniques were studied: surface topology air-gap reluctance system (STARS), residual magnetic flux leakage (RMFL) and stray magnetic flux leakage (SMFL). Numerical results showed that in the case of the STARS and SMFL, defect signatures in signals were observed for investigated near-side defects, but not for far-side defects. The signature of the far-side defect in the RMFL was observed, however its peak-to-peak value was only about $8 \%$ of the value corresponding to the near-side defect.
\end{abstract}

Keywords Magnetic flux leakage $\cdot$ Near-side defect $\cdot$ Far-side defect $\cdot$ Finite element method

\section{Introduction}

Many storage and transmission structures, such as aboveground storage tanks (AST) and transmission pipelines, are made of a ferromagnetic steel. Unfortunately, ferrite based steel grades are prone to corrosion that can lead to malfunction of the mentioned structures. On the other hand, ferromagnetic properties of these steel grades enable to use magnetic NDT methods to inspect integrity of the structures made of them. One of these methods is magnetic flux leakage (MFL), which is commonly used for inspection of AST floors and natural gas pipelines. This method in its classic form is classified as an active one, because an object under investigation is magnetized by the source of a static magnetic field. Although electromagnets can be used as such a source, most practical implementations of the MFL are based on permanent magnets. Advantage of the latter approach is

Zbigniew Usarek

zbigniew.usarek@pg.edu.pl

1 Advanced Materials Center, Gdańsk University of Technology, ul. Narutowicza 11/12, 80-233 Gdańsk, Poland

2 Institute of Nanotechnology and Materials Science, Faculty of Applied Physics and Mathematics, Gdańsk University of Technology, ul. Narutowicza 11/12, 80-233 Gdańsk, Poland that it does not require an additional power supply, which is of great importance when the inspection is made by the battery supplied device. The example of such a device is an MFL pipeline inspection gauge (PIG) which is commonly used for an in-line inspection of crude oil and natural gas pipelines. Although the MFL-PIG inspects a pipeline wall condition from the pipeline interior, it enables to detect defects located both on the inside and outside surface of the wall.

Locations of the wall defects are not the only information which can be derived from the MFL signal. A properly processed MFL signal can also be used for both classification of defects (e.g. as cracks, dents, metal losses due to corrosion) and for their size determination as well. However, reliability of the latter procedure can be reduced, if the location of a defect on one of the two sides of the wall is not explicitly determined.

Terminology used in the MFL related publications to name these two types of defects is inconsistent. This is mainly due to various geometries of objects under consideration in various MFL studies. The knowledge about diversity of the nomenclature regarding the near- and far-side defects is necessary for performing a comprehensive query in this field. In order to systematize the terminology, various terms that describe differently located defects are collected in Table 1. Among many listed variations the terms 'near-side' and 'far- 
Table 1 Summary of various terms for opposite located defects that appear in the MFL related literature

\begin{tabular}{|c|c|c|}
\hline Location of defects & $\begin{array}{l}\text { Surface near the } \\
\text { sensor }\end{array}$ & $\begin{array}{l}\text { Surface far from the } \\
\text { sensor }\end{array}$ \\
\hline $\begin{array}{l}\text { Terms describing } \\
\text { defects }\end{array}$ & $\begin{array}{l}\text { internal }[1-7] \\
\text { inner/inside }[8,9] \text {, } \\
\text { near-side/surface } \\
\quad[10-13], \\
\text { top [14-16], } \\
\text { front [17], } \\
\text { front-side }[18,19]\end{array}$ & $\begin{array}{l}\text { external [1-7], } \\
\text { outer/outside [8, } \\
20], \\
\text { far-side/surface } \\
\text { [10-13, 21], } \\
\text { bottom [14-16, 22], } \\
\text { back [17], } \\
\text { back-side [18, 19, } \\
\text { 23-26], } \\
\text { opposite-side [27], } \\
\text { sub-surface [28] }\end{array}$ \\
\hline
\end{tabular}

side' are probably most unequivocal and universal, so these two terms are generally used in the rest of this article.

Disclosure of a defect in the MFL method is possible because its presence leads to a deflection of the magnetic flux in its vicinity. This in turn leads to a partial leakage of the magnetic flux that can be measured on both sides of the wall. Suppose there are two identical surface-breaking defects differing only in their location relative to an MFL tool. In both cases different MFL signals can be expected. There are at least two factors that contribute to this difference in the signals. The first one is the distance between the MFL sensors and a defect, which is greater for the far-side defect than for the near-side defect. The second one is the presence of a ferromagnetic wall in the case of the far-side defect or its absence in the case of the near-side defect. As the distance between the MFL sensors and the defect increases, the measured signal generally weakens. On the other hand, as reported by Wu et al. [29] the presence of the ferromagnetic wall between the MFL sensors and the defect makes the signal stronger than if there is air instead of the ferromagnetic wall. The final difference between MFL signals for identical near- and far-side defects depends on the aforementioned factors as well as on the geometry of the defects themselves. As observed by Romero-Ramirez et al. [14], defects of the same geometry located either on the top (near-side) or bottom (farside) surface of the steel plate can produce very similar MFL signals. Usually, however, the signal from the far-side defect is weaker than the signal from the near-side defect of identical shape and size. Results of a numerical analysis carried out by Sorabh et al. [1] confirm this statement for the same $50 \%$ metal losses located on the opposite sides of the wall. Therefore, one can claim that there is a risk of underestimation of defect dimensions, especially its depth, in situation when a far-side defect is classified as a near-side one. In this paper, the aforementioned claim was verified.

The second section discusses methods that can help in unambiguous determination of the defect location on the basis of juxtaposing the MFL signal with the signal measured by one of these methods. Samples from previous studies and a ready-made MFL measuring system, which was modified for the purposes of this study, were used in the experiment. Details of the measuring stand are described in Sect. 3. The aim of the experiment was to validate the results obtained by simulation. Section 4 describes the models developed for simulation using the finite element method (FEM). Section 5 contains the results of both the experiment and the simulation. Section 6 contains the conclusions drawn from them.

Two series of simulations were performed. The aim of the first one, in which cuboidal defects were simulated, was to compare its results with the experimental ones. The aim of the second, more important series of simulations was to test the ability to distinguish near- and far-side defects by the three considered methods in a situation where the MFL signal of both defects is practically indistinguishable. This is the first such comparison of various magnetic methods capable of distinguishing near- from far-side defects, especially in the context of their use in parallel with the measurement of classic MFL. It is also new to show that two different semielliptical defects, often encountered in practice, can generate a very similar MFL signal.

\section{Magnetic Methods of Distinction Between Near- and Far-Side Defects}

\subsection{Surface Topology Air-Gap Reluctance System (STARS)}

When an MFL magnetizing unit scans the near surface, an air-gap between a pole piece and the surface changes its width in presence of a near-side defect. Appearance of the defect results in increase of the air-gap reluctance and in decrease of magnetic flux density. In the presence of a far-side defect no significant change of the reluctance is registered. An application of the mentioned phenomena is described in $[15,30]$. The setup that utilizes this technology is called the surface topology air-gap reluctance system (STARS) and is currently patented [30]. With this property, the STARS in combination with the classic MFL unit is a promising candidate for the construction of a tool, which is able to distinguish near-side defects from far-side defects. Its relatively simple adaptation was used in the presented study to measure the air-gap magnetic field changes due to near- and far-side defects.

\subsection{Weak Magnetic Flux Leakage (WMFL)}

In the classic MFL technique it is usually desirable to magnetize the wall with a sufficiently strong magnetic field what ensures homogenous magnetization of the wall cross-section. This approach also increases the probability of detection 
(POD) of far-side defects. In some cases, however, a low strength magnetization of the wall offers brand new capabilities in comparison to classic MFL.

\subsubsection{Low Frequency AC Magnetization}

In [31] Cheng proposed a method of inspection of the far side wall-thinning with the use of very low strength magnetization MFL testing. An electromagnet was used to magnetize a test object. Cheng analyzed a DC and very low AC excitation of the electromagnet. It was observed that the quantity called differential magnetic-leaking reluctivity is a linear function of remaining wall thickness. Another example of a research, where a low frequency $\mathrm{AC}$ excitation is used to investigate near- and far-side defects is presented by Zaini et al. [19]. The authors used a differential probe consisted of two anisotropic magneto-resistive (AMR) sensors to measure an MFL signal generated by near and far-side slits. As reported, the imaginary part of the signal measured at the location of a near-side slit is inverted compared to the signal measured for a far-side slit. The difference between both signal patterns is very clear, so it seems to be a very promising way of distinction between near- and far-side defects. Comparable results were obtained earlier by Tsukada et al., who used similar setup to detect inner cracks in thick steel plates [9].

\subsubsection{Weak Magnetization}

Application of a weak magnetization leads to unique patterns of a biaxial MFL signal as is shown by the results presented in [17]. In this case authors applied a DC current to an electromagnet to achieve a magnetic flux density of $0.6 \mathrm{~T}$ inside the specimen. They used three-step post-processing procedure that led to evaluation of defect dimensions. Every step relied on an artificial neural network (ANN) designed for a certain task. Input to each ANN consisted of parameters describing functions that approximate a biaxial MFL signal. The first ANN, called classification-NN, was responsible for classification of the MFL signal section as defective or not. The second one, called localization-NN, performed classification of a defect as located either on the front or on the back surface. The third one, called identification-NN, evaluated the depth and the width of a defect. Similar approach that is based on another machine learning method of classification-support vector machine (SVM) — was described in [4]. A measurement technique called weak magnetic flux leakage (WMFL) was proposed by Liu et al. as potential method for studying differences between near-side and far-side defects [8]. And indeed one can observe clear qualitative differences between the WMFL signals measured for near- and far-side defects, especially in the case of the radial component.

\subsubsection{Stray Magnetic Flux Leakage (SMFL)}

One of the criteria for selecting measurement methods for the study described in this paper was the possibility of a simple expansion of the classic MFL unit with additional sensors allowing for an unambiguous distinction between nearand far-side defects. In this context, the disadvantage of the approaches described in the two previous subsections is the necessity to use an additional source of a weak (DC or AC) magnetic field. There arose a question, how to achieve weak magnetization without introducing an additional source of a magnetizing field. The answer for this question is utilization of the magnetic flux that normally leaks from a magnetic circuit consisting of a magnetizer and an inspected object. Such magnetic circuit is not ideal, i.e. fully closed. Part of the flux, which can be called the stray flux, creates closed paths outside the magnetic circuit. The stray flux is present both at the front and back of the MFL magnetizer. It results in non-homogenous magnetization of an object both in the horizontal as well as vertical direction in relation to the object wall. The resulting magnetization is weaker than between the poles of the magnetizer and theoretically leads to different MFL signals measured for near- and far-side defects. The proposed name of this variation of the WMFL is the stray magnetic flux leakage (SMFL). It is worth noting that the abbreviation SMFL is not unique, because it can also refer to the SpirALL MFL technology of TD Williamson or selfmagnetic flux leakage [32]. Nonetheless, for the sake of this paper the abbreviation SMFL is referred to as the stray magnetic flux leakage.

\subsection{Residual Magnetic Flux Leakage}

A measurement technique called residual magnetic flux leakage (RMFL) was proposed by Babbar and Clapham [33] as an alternative method for studying defects in a pipeline wall. They compared experimental results obtained with the help of the active and residual MFL for three artificial defects with different geometries. Two types of the magnetizer movement were also investigated. These two types of the movement resulted in different distribution of residual magnetization, and thus the observed RMFL pattern. The first type concerned the situation when the magnetizer moved away perpendicularly to the wall surface (perpendicular lift-off). The second type applied to the situation, when the magnetizer moved parallel to the wall surface. In both situations the RMFL was measured after removal of the magnetizer from the vicinity of a defect. It is worth mentioning that the second situation better corresponds to a movement of most MFL devices. It should be mentioned that Babbar and Clapham did not prove utility of the RMFL in the context of distinction between near-side and far-side defects. Being so, it was decided to 

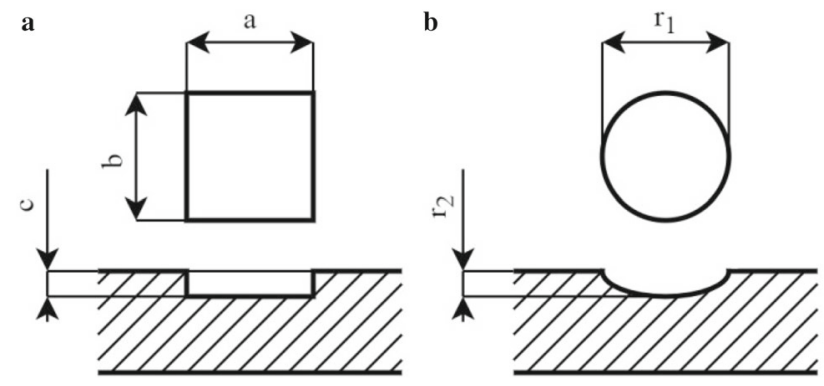

Fig. 1 Geometries and parameters describing investigated defects, which took the form of $\mathbf{a}$ rectangular loss or $\mathbf{b}$ corrosion pit with a semi-elliptical cross-section

investigate this capability of the RMFL signal in the present study.

\subsection{Investigated Techniques}

Some of techniques listed so far in the Sect. 2 undoubtedly have a potential to discriminate between near- and far-side defects. However, they have also some limitations. Most of them cannot be applied as a complementary method that aids the traditional MFL, because they usually require an additional, power-consuming magnetizing unit. In practice it can be economically unreasonable. That is why in this study we focused only on methods that do not need any additional source of a magnetizing field, i.e. STARS, SMFL and RMFL.

\section{Samples and Experimental Setup}

Experiment was carried out for three steel plates made of the 18G2A (S355 equivalent) grade. The plates were made of an $8 \mathrm{~mm}$ thick steel sheet. They were $292 \mathrm{~mm}$ long and $100 \mathrm{~mm}$ wide. Rectangular defects were milled in the plates symmetrically to their length and width. Each plate had one such defect. All defects had the same width $b$ equal to $10 \mathrm{~mm}$ and the comparable depth $c$ of about $2 \mathrm{~mm}$. The difference between defects was their length $a$, which was equal to 10 , 15 or $24 \mathrm{~mm}$. Definitions of aforementioned dimensions are presented in Fig. 1a.

Two N42 neodymium permanent magnets with dimensions $50 \times 50 \times 25 \mathrm{~mm}$ were used in the magnetizer. A distance covered by the MFL unit was measured with the help of a digital encoder. All signals were transmitted to the NI USB-6009 board and then to the PC in order to perform post-processing procedures in the LabVIEW environment. The complete experimental setup is depicted in Fig. 2.

Figure 3 contains the definitions of the most important dimensions related to the location of the sensors used in the research. The classic MFL signal was measured using the probe consisting of two Hall-effect sensors SS495A. The

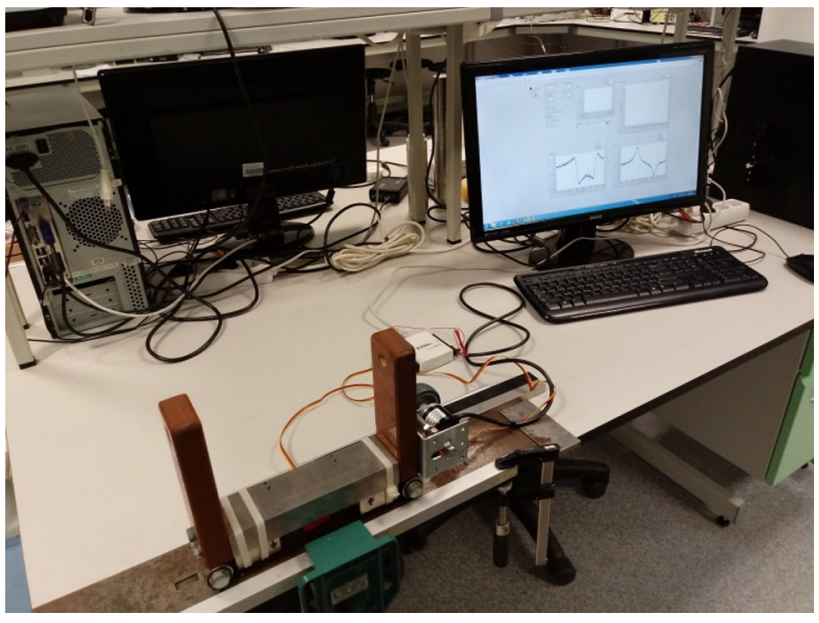

Fig. 2 The setup used in the experiment. In the foreground one can see the MFL unit placed on two adjacent steel plates. In the background one can see the screen displaying the front panel of a LabVIEW virtual instrument

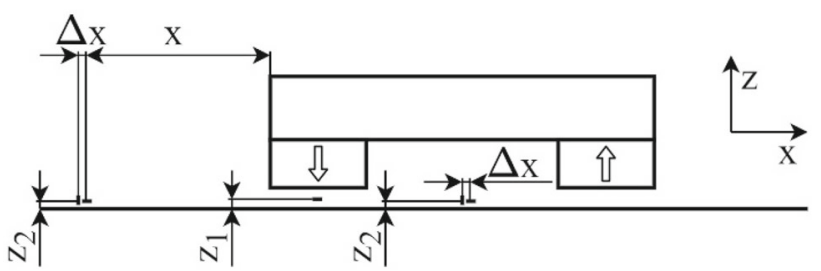

Fig. 3 A diagram showing the most important distances related to the location of the sensors

first sensor measured $B_{x}$ component of the magnetic field, i.e. the component parallel to the movement direction of the magnetizer. This sensor was followed by the second sensor measuring $B_{z}$ component, which is perpendicular to the scanned surface. Spacing between these two sensors $\Delta x=$ $3 \mathrm{~mm}$ and their lift-off $z_{2}=2 \mathrm{~mm}$.

The pocket size smart magnetic sensor SMS-102 manufactured by Asonik was used to perform measurements of the magnetic field in the air-gap, because it has a measurement range of $\pm 1999.9 \mathrm{mT}$. Such range was sufficient to capture the air-gap field changes and simultaneously avoid saturation of the hall sensor due to the field close to $1 \mathrm{~T}$. The end of the probe containing the hall sensor was placed under the back pole of the magnetizer. The lift-off $z_{1}$ of the STARS probe was equal to $5 \mathrm{~mm}$.

SMFL was measured at the distance $x=90 \mathrm{~mm}$ behind the back-pole in the case of $B_{z}$ component. The component $B_{x}$ was measured at the distance $x+\Delta x$ behind the backpole. Two Hall-effect sensors A1324 were used to measure those components and both of them had the same lift-off $z_{2}$ $=2 \mathrm{~mm}$. An additional plate was placed after the currently investigated plate allowing a free passage of the magnetizer while performing STARS and SMFL measurements. 


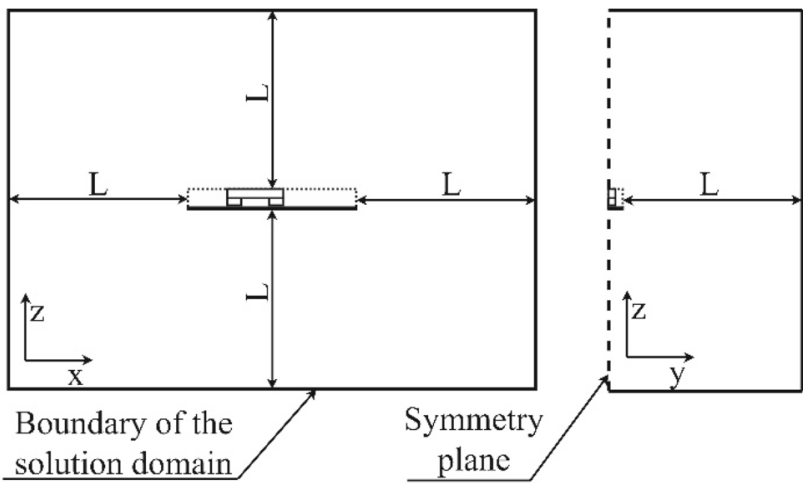

Fig. 4 The background box and its relationship with the parameter L. Side and front view of the box are presented on the left and on the right respectively

In this study, RMFL signals were registered with the use of the same probe as in the case of SMFL measurements. Measured RMFL signals exhibit lower signal-to-noise ratio (SNR), and thus they were post-processed using a forward-backward digital filter in order to reduce noise without introducing a phase shift to a filtered signal.

\section{Finite Element Analysis}

All simulations were carried out using the FEM. The project was created in Maxwell 3D, a part of the ANSYS Electronics Suite 18.2. The adaptive mesh refinement was applied to obtain results with a desired accuracy. Dimensions of the background box, presented in Fig. 4, were determined by performing sensitivity analysis of the MFL solution. The goal of this analysis was to achieve the minimal relative change of the MFL peak-to-peak value less than $1 \%$. It was determined that the distance $L$, i.e. the minimal distance of model components from outer walls of the box should be greater than or equal to $640 \mathrm{~mm}$. One symmetry plane was defined in the project, i.e. $y=0$. On the outer walls the Neumann boundary conditions were defined, which means that $\mathbf{H}$ was tangential to the boundary. The project was divided into four designs regarding to the investigated methods, i.e. classic MFL, STARS, SMFL, and RMFL. Except the RMFL, each design consisted of a series of magnetostatic simulations performed for different positions of the magnetizer in relation to the steel plate, which reflects the actual MFL measurement. In the mentioned simulations the magnetizer was moved in $2 \mathrm{~mm}$ steps. Geometries of simulated defects took one of the forms presented in Fig. 1, i.e. either the rectangular loss or the corrosion pit.

\subsection{Simulation of the Classic MFL Setup}

The geometry used in the classic MFL design is presented in Fig. 5. Magnetic properties of the material assigned to the

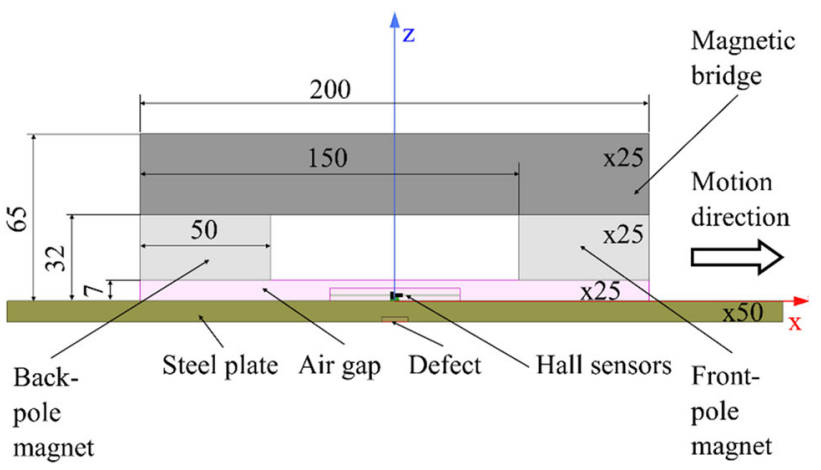

Fig. 5 Geometry of the classic MFL design. Each solid included in the design is shown

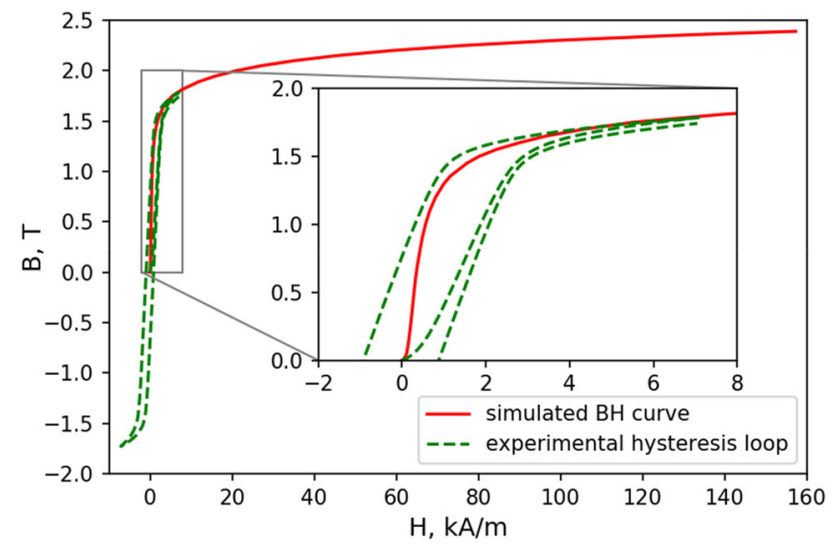

Fig. 6 The relationship between $B$ and $H$ used for simulation of magnetic properties of magnetic bridge and steel plate. Additionally, for the sake of comparison, an example of a hysteresis loop measured for a sample made of $18 \mathrm{G} 2 \mathrm{~A}$ steel (of which the steel plate is also made) is presented

magnetic bridge as well as to the steel plate were defined by a non-linear $B$ - $H$ curve presented in Fig. 6 . This curve referred to the low carbon steel SAE 1020. The reason for using this curve instead of experimentally measured is the fact that in the area of the plate between the poles of the magnetizer, the values of the magnetic field strength $H$ can reach tens of $\mathrm{kA} / \mathrm{m}$. So high values of $H$ were impossible to achieve using the hysteresis loop measurement system available in the authors' laboratory. In the near saturation region (above $4 \mathrm{kA} / \mathrm{m}$ ), the simulated $B-H$ relationship is close to the $B-H$ curve of the material used in the experiment. A significant difference between the curve used in the simulations and the exemplary experimental curve can be observed for $H$ below $4 \mathrm{kA} / \mathrm{m}$. This can result in the difference between the experiment and simulation results over areas of the plate with lower magnetization. As the material of both magnets the Arnold Magnetics N42-20C was selected from the Maxwell material library. All the remaining solids, including background, were treated as the air. 


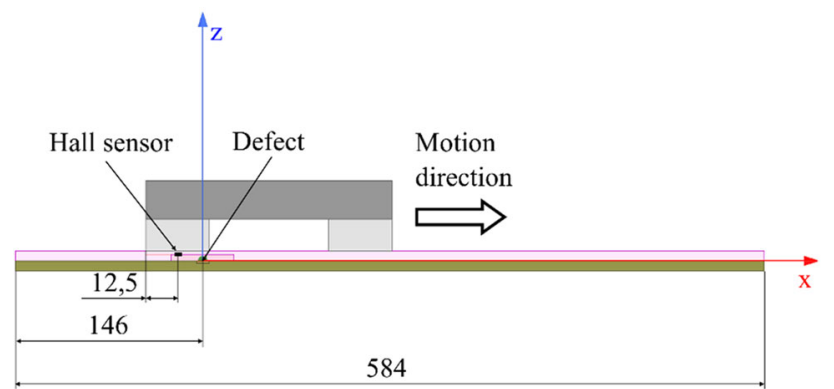

Fig. 7 Geometry used in the STARS design. Most dimensions are identical to the MFL design. Only those characteristic of the STARS project are given

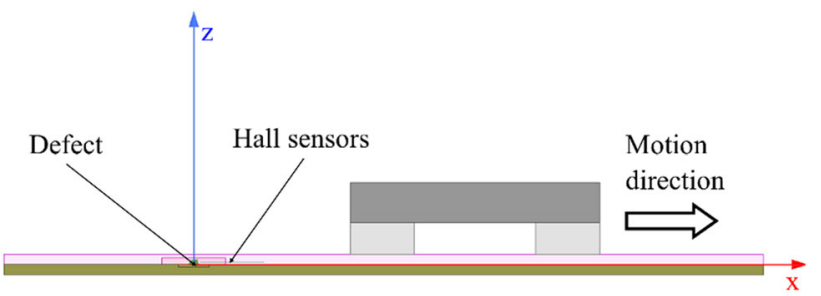

Fig. 8 Geometry used in the SMFL design. All dimensions are identical to the STARS design. The position of the SFML sensors is the same as in the experiment

\subsection{Simulation of the STARS Setup}

The STARS signal was measured at the location under the back-pole magnet, in the middle of the magnet length, and at the $5 \mathrm{~mm}$ lift-off as presented in Fig. 7. In this case the length of the plate is twice the length of the plate simulated in the classic MFL design, which reflects measurement conditions during the experiment.

\subsection{Simulation of the SMFL Setup}

Geometry used in the SMFL is very similar to that used in the STARS design. The only difference concerns the location of the SMFL sensors. Points, where two components of the SMFL, i.e. $B_{x}$ and $B_{z}$ were measured, are located respectively 93 and $90 \mathrm{~mm}$ from the back-pole end as presented in Fig. 8. The lift-off value equals to $2 \mathrm{~mm}$.

\subsection{Simulation of the RMFL}

In comparison to the previous designs, the RMFL design was characterized by a simpler geometry, because it does not consist of the magnetizer, as can be seen in Fig. 9. For a particular defect the RMFL distribution was determined based on one simulation only. The distribution was determined along the measurement path at the lift-off equal to $2 \mathrm{~mm}$. Remanent magnetization of the steel plate was introduced by defining the coercive force $H_{c}$ as equal to $-900 \mathrm{~A} / \mathrm{m}$. The given $H_{c}$

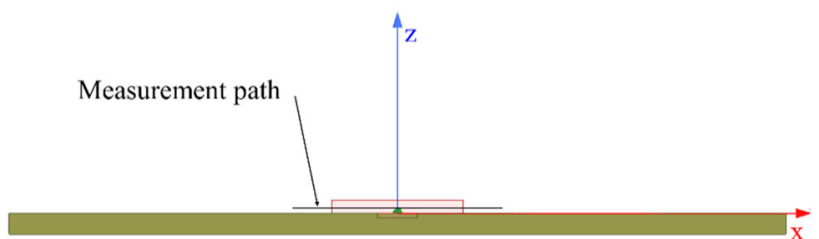

Fig. 9 Geometry used in the RMFL design. The location of the measurement path is also indicated

value is the average value derived from hysteresis loop measurements of samples made of the same steel grade as the plate.

\section{Results and Discussion}

Figure 10 shows results from the FEA obtained for a rectangular defect with the length $a=10 \mathrm{~mm}$ and depth $c=$ $1.7 \mathrm{~mm}$ located either on the near- or far-side surface. In this particular case, defect signals of both MFL components for the far-side defect are clearly weaker than corresponding defect signals generated by the near-side defect. The defect signal of the STARS appears only for the near-side defect. A similar effect can be observed for the both SMFL components. Defect signals of the raw RMFL are not so clear due to demagnetization of the plate resulting in a large bias of $B_{x}$ and inclination of $B_{z}$. Therefore, Fig. 10 shows the RMFL signal without background associated with the demagnetization of the plate. Also in this case, a much higher amplitude of the near-side defect signal can be observed compared to the far-side defect.

Experimental measurements were carried out for the defect of the same geometry, i.e. $a=10 \mathrm{~mm}, b=10 \mathrm{~mm}, c$ $=1.7 \mathrm{~mm}$, in order to validate simulation models. Results of these measurements are presented in Fig. 11. Defect signals of the experimental MFL are about twice weaker in amplitude than corresponding defect signals determined via simulation. Regardless of this difference, the far- to near-side ratios of the MFL peak-to-peak values are similar for simulation and experimental results. The experimental results of the STARS signal are close to the corresponding results presented in Fig. 10. One can observe the difference in background for the near- and far-side defect. It is mainly associated with slightly different gaps (due to imperfect edge matching between the plates) between two adjacent plates, which highly influence reluctance of the whole magnetic circuit. Increased reluctance due to the mentioned gap generally leads to decrease in magnetic field measured under the back pole. Close similarity of the experimental and simulation results is observed for the SMFL. The experimental RMFL signal, similarly as previously presented FEA results, was processed by subtracting the background associated with demagnetization. 

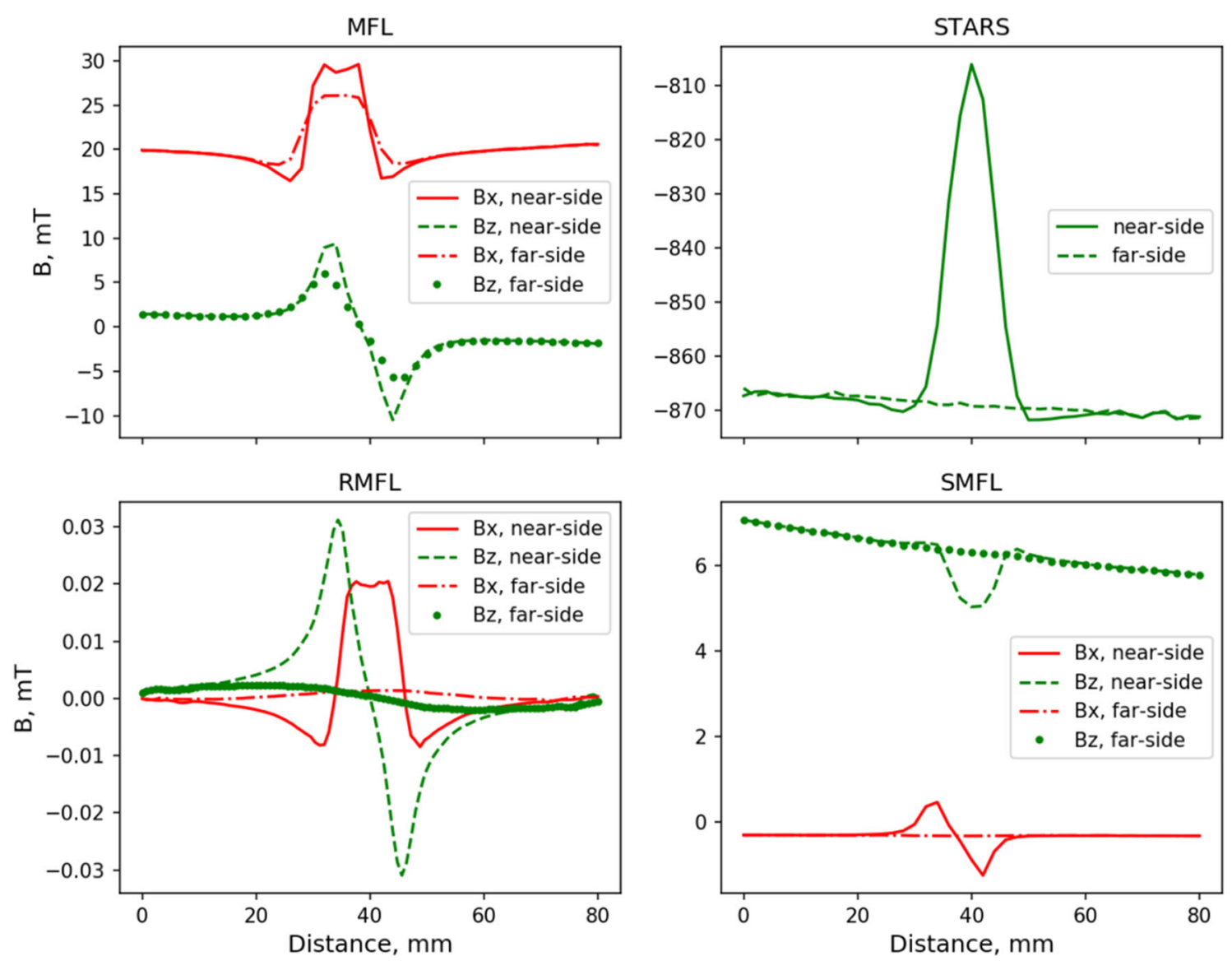

Fig. 10 FEA based comparison of the investigated techniques. Presented results were obtained for the $10 \mathrm{~mm}$ long defect with depth of $1.7 \mathrm{~mm}$ located either on the near- or far-side surface

Unambiguous defect signal was registered via RMFL measurements only for the near-side defect. It is noticeable that the amplitude of the defect signal determined on the basis of the experiment is several times higher than in the case of a similar signal determined by the simulation. It can result from a higher than assumed magnetization of the experimentally investigated plate.

Corrosion pits, which are the most common type of corrosion defects in transmission pipelines [6], can be better simulated using the semi-elliptical geometry [7] depicted in Fig. 1b than one in Fig. 1a. That is why this kind of geometry was chosen to make a comparison of the investigated techniques. Parameters of the reference near-side defect was assumed as follows: $r_{1}=5 \mathrm{~mm}, r_{2}=2 \mathrm{~mm}$. Optimization analysis was performed in order to determine $r_{1}$ and $r_{2}$ values of such far-side defect, that generates the MFL signal similar to one generated by the reference defect. In this way, the parameters of the far-side defect were determined as follows: $r_{1}=5 \mathrm{~mm}, r_{2}=2.8 \mathrm{~mm}$. Figure 12 shows a comparison of FEA results obtained for the reference defect with those obtained for the aforementioned far-side defect. In order to better visualize defect signals of the RMFL com- ponents, background generated via simulation of the plate with no defects was subtracted from the raw RMFL signal. It can be seen that the MFL signals of both defects are very similar to each other. In order to quantify this similarity, one can calculate the relative difference between peak-to-peak

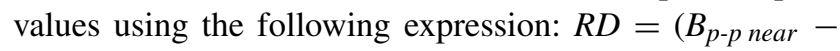
$\left.B_{p-p \text { far }}\right) / B_{p-p \text { near }} \times 100 \%$. So calculated difference is $1.0 \%$ in the case of $B_{x}$ and $0.8 \%$ in the case of $B_{z}$. In the analyzed example, the far-side defect had the depth by $10 \%$ of wall thickness greater than the near-side defect. This comparison shows that a far-side defect with a significantly greater depth can produce an MFL signal similar to one generated by a near-side defect. However, results presented in Fig. 12 show also that each investigated complementary technique enables to distinguish the near-side defect from the far-side one. For the STARS and SMFL results, defect signals appear only for the near-side defect $(R D=100 \%)$. In the case of the RMFL signal, weak defect signals for the far-side defect can be also seen. The relative difference $R D$ between the RMFL defect signals for differently located defects is therefore slightly less than 100\%: $92.5 \%$ in the case of $B_{x}$ and $90.6 \%$ in the case of $B_{z}$. 

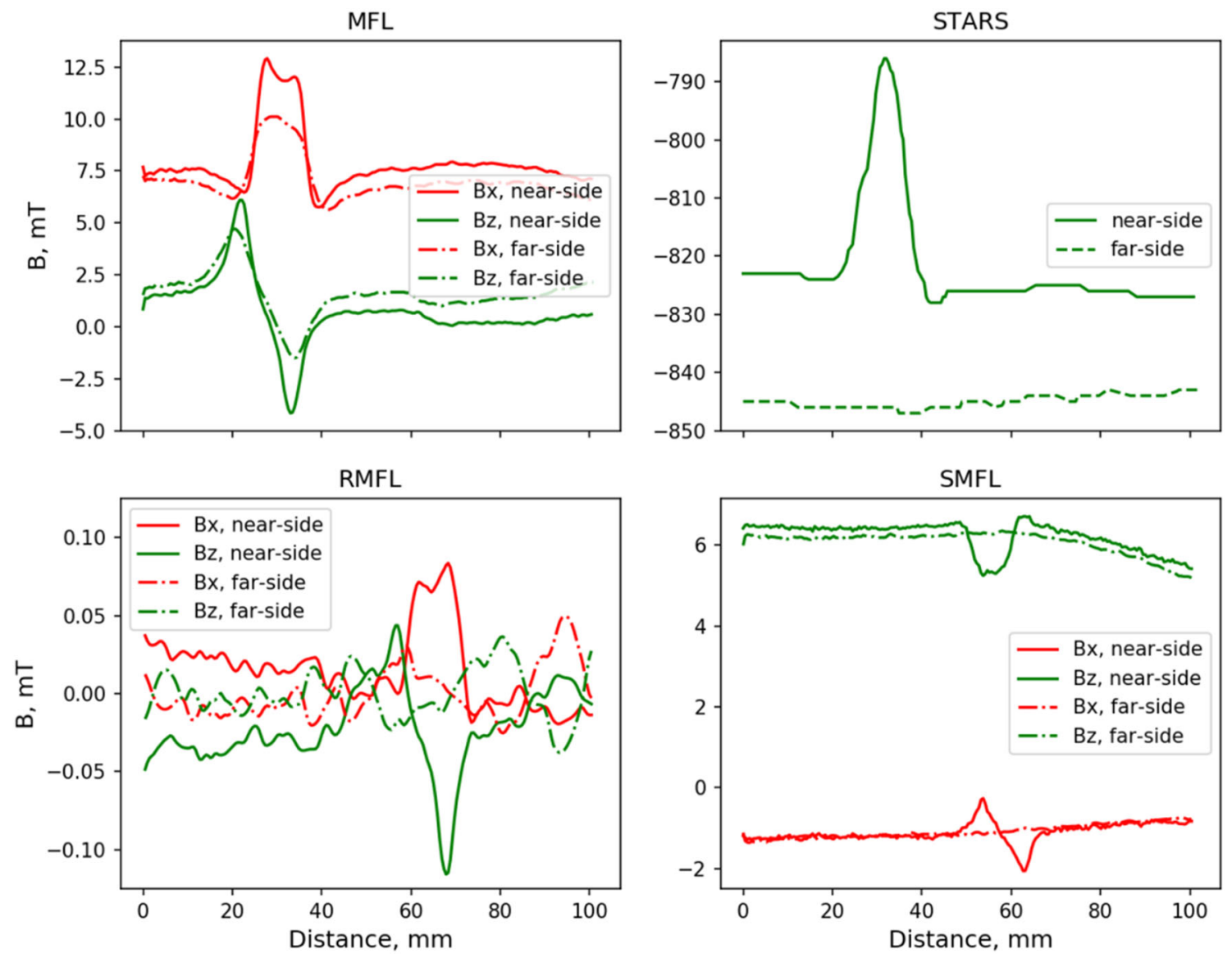

Fig. 11 Comparison of the investigated techniques based on the experimental results, that were obtained for the $10 \mathrm{~mm}$ long defect located either on the near- or far-side surface

\section{Conclusions}

For the purpose of this study, the existing MFL measurement system and a sample with a rectangular defect were adapted to the preparation of the initial series of simulations and the experiment to validate it. High quantitative and qualitative agreement of SMFL signals obtained by the simulations and experiment was observed. In the case of MFL results, different levels of the $B_{x}$ component background can be observed and the defect signals measured experimentally are almost two times smaller than simulated ones. However, both types of results are characterized by qualitative agreement. In the case of the experimental STARS results, a shift in the background level can be noticed, which results from the rotation of the test plate between these two measurements and the change in the STARS measurement conditions. There was also a large discrepancy between the RMFL simulation results and the experimental RMFL measurement results. In order to increase the compatibility of the results of similar FEM simulations with the experiment in the future, direct measurements of B-H curves for the simulated materials should be performed.
Most important part of this study is associated with the results of second series of simulations, in which two semi-elliptical defects were investigated. Results of these simulations show that all three investigated techniques, i.e. STARS, RMFL and SMFL, have a capability to discriminate between opposite defects that give similar MFL signals. However, all these techniques have some disadvantages. As observed for the experimental results, the RMFL is characterized by a small defect signal, and thus it can be useless for discrimination of relatively shallow defects. As noticed by Babbar and Clapham, the RMFL " (...) technique involves the use of sensitive probes to detect the flux leakage signals, which have about one tenth of the strength of the active flux leakage commonly used" [33]. It is possible to use more sensitive probes based on AMR or giant magnetoresistive (GMR) sensors to perform RMFL measurements.. An attribute of the STARS, which can be a limitation of this technique, is the need of a sensor with a relatively wide range of measured magnetic field values. In this research these values were about $0.8 \mathrm{~T}$. Most linear Hall-effect sensors available on the market offer a maximum linear range of the measured magnetic field that usually does not exceed \pm 100 

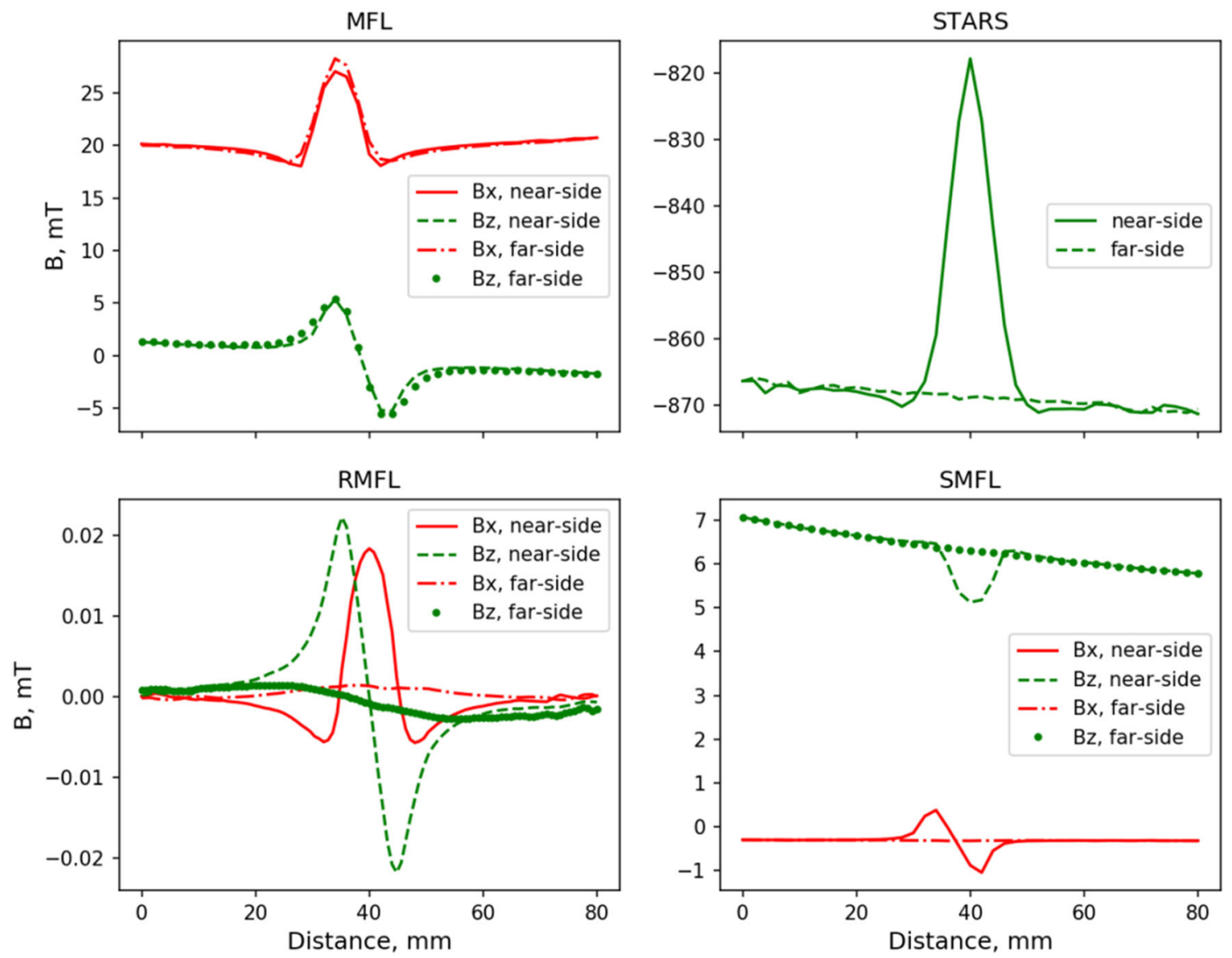

Fig. 12 FEA based comparison of the investigated techniques. Presented results were obtained for two corrosion pits with the radius $r_{l}=$ $5 \mathrm{~mm}$. The near-side pit had the radius $r_{2}=2 \mathrm{~mm}$, while the far-side

one had the radius $r_{2}=2.8 \mathrm{~mm}$. The background was subtracted from the raw RMFL to increase visibility of its defect signal

mT (e.g. AH49E, SS39ET), so they are not able to measure magnetic fields close to $1 \mathrm{~T}$. The third of the considered methods, i.e. SMFL, is characterized by defect signals with a slightly smaller amplitude than the signals measured by MFL sensors. Sensors with similar parameters as in the case of the classic MFL can be used to measure the SMFL signal. The $B_{z}$ component of the SMFL signal is characterized by a high background value, which is associated with two disadvantages. The first is that the sensors risk going beyond their linear operating range. The second one is that a high background value forces an increase in the voltage range measured at the input of the $\mathrm{AD}$ converter. This in turn reduces the precision of SMFL measurements. The problem described does not exist for the $B_{x}$ component. Due to its simple implementation and potentially high efficiency in distinguishing between near-side and far-side defects, future research by the authors will be devoted to the properties of the SMFL signal.

Acknowledgements Calculations were carried out using the software provided by the Academic Computer Center in Gdańsk.
Open Access This article is licensed under a Creative Commons Attribution 4.0 International License, which permits use, sharing, adaptation, distribution and reproduction in any medium or format, as long as you give appropriate credit to the original author(s) and the source, provide a link to the Creative Commons licence, and indicate if changes were made. The images or other third party material in this article are included in the article's Creative Commons licence, unless indicated otherwise in a credit line to the material. If material is not included in the article's Creative Commons licence and your intended use is not permitted by statutory regulation or exceeds the permitted use, you will need to obtain permission directly from the copyright holder. To view a copy of this licence, visit http://creativecomm ons.org/licenses/by/4.0/.

\section{References}

1. Sorabh, G.A., Chandrasekaran, K.: Finite element modeling of magnetic flux leakage from metal loss defects in steel pipeline. J. Fail. Anal. Prev. 16, 316-323 (2016). https://doi.org/10.1007/ s11668-016-0073-6

2. Kopp, G., Willems, H.: Sizing limits of metal loss anomalies using tri-axial MFL measurements: a model study. NDT E Int. 55, 75-81 (2013). https://doi.org/10.1016/j.ndteint.2013.01.011 
3. Yilai, M., Li, L.: Research on internal and external defect identification of drill pipe based on weak magnetic inspection. Insight Non-Destr. Test. Cond. Monit. 56, 31-34 (2014). https://doi.org/ 10.1784/insi.2014.56.1.31

4. Jian, Q., Ai, Z.: Internal and external defect identification of pipelines using the PSO-SVM method. Insight Non-Destr. Test. Cond. Monit. 57, 85-91 (2015). https://doi.org/10.1784/insi.2014. 57.2.85

5. Jiao, J., Chang, Y., Li, G., He, C., Wu, B.: Study on low frequency AC magnetic flux leakage detection for internal and external cracks of ferromagnetic structures. Yi Qi Yi Biao Xue Bao/Chin. J. Sci. Instrum. 37, 1808-1817 (2016)

6. Vanaei, H.R., Eslami, A., Egbewande, A.: A review on pipeline corrosion, in-line inspection (ILI), and corrosion growth rate models. Int. J. Press. Vessel. Pip. 149, 43-54 (2017). https://doi.org/10. 1016/j.ijpvp.2016.11.007

7. Pluvinage, G., Bouledroua, O., Hadj Meliani, M., Suleiman, R.: Corrosion defect analysis using domain failure assessment diagram. Int. J. Press. Vessel. Pip. 165, 126-134 (2018). https://doi. org/10.1016/j.ijpvp.2018.06.005

8. Liu, B., Cao, Y., Zhang, H., Lin, Y.R., Sun, W.R., Xu, B.: Weak magnetic flux leakage: a possible method for studying pipeline defects located either inside or outside the structures. NDT E Int. 74, 81-86 (2015). https://doi.org/10.1016/j.ndteint.2015.05.008

9. Tsukada, K., Majima, Y., Nakamura, Y., Yasugi, T., Song, N., Sakai, K., Kiwa, T.: Detection of inner cracks in thick steel plates using unsaturated $\mathrm{AC}$ magnetic flux leakage testing with a magnetic resistance gradiometer. IEEE Trans. Magn. (2017). https://doi.org/10. 1109/TMAG.2017.2713880

10. Atherton, D.L., Daly, M.G.: Finite element calculation of magnetic flux leakage detector signals. NDT Int. 20, 235-238 (1987). https:// doi.org/10.1016/0308-9126(87)90247-1

11. Pham, H.Q., Trinh, Q.T., Doan, D.T., Tran, Q.H.: Importance of magnetizing field on magnetic flux leakage signal of defects. IEEE Trans. Magn. (2018). https://doi.org/10.1109/TMAG.2018. 2809671

12. Singh, W.S., Rao, B.P.C., Vaidyanathan, S., Jayakumar, T., Raj, B.: Detection of leakage magnetic flux from near-side and farside defects in carbon steel plates using a giant magneto-resistive sensor. Meas. Sci. Technol. 19, 015702 (2008). https://doi.org/10. 1088/0957-0233/19/1/015702

13. Pullen, A.L., Charlton, P.C., Pearson, N.R., Whitehead, N.J.: Practical evaluation of velocity effects on the magnetic flux leakage technique for storage tank inspection. Insight Non-Destr. Test. Cond. Monit. 62, 73-80 (2020). https://doi.org/10.1784/insi.2020. 62.2 .73

14. Romero Ramírez, A., Mason, J.S.D., Pearson, N.: Experimental study to differentiate between top and bottom defects for MFL tank floor inspections. NDT E Int. 42, 16-21 (2009). https://doi. org/10.1016/j.ndteint.2008.08.005

15. Jim, C., Pearson, N., Boat, M.: Capability of modern tank floor scanning with magnetic flux leakage. In: Proceedings of the 19th World Conference on Non-Destructive Test. Vol 2016, pp. 1-11 (2016)

16. Pullen, A.L., Charlton, P.C., Pearson, N.R., Whitehead, N.J.: Magnetic flux leakage scanning velocities for tank floor inspection. IEEE Trans. Magn. 54, 1-8 (2018). https://doi.org/10.1109/ TMAG.2018.2853117

17. Abe, M., Biwa, S., Matsumoto, E.: Evaluation of surface flaw by magnetic flux leakage testing using amorphous MI sensor and neural network. In: Lecture Notes in Electrical Engineering. pp. 15-33. Springer, Berlin, Heidelberg (2009)

18. Hadi Putera Zaini, M.A., Mawardi Saari, M., Nadzri, N.A., Mohd Halil, A., Tsukada, K.: An MFL probe using shiftable magnetization angle for front and back side crack evaluation. In: Proceedings of the 2019 IEEE 15th International Colloquium on Signal Processing \& Its Applications (CSPA). pp. 157-161. IEEE (2019)

19. Zaini, M.A.H.P., Saari, M.M., Nadzri, N.A., Halil, A.M., Hanifah, A.J.S., Ishak, M.: Effect of excitation frequency on magnetic response induced by front- and back-side slits measured by a differential AMR Sensor probe. In: Lecture Notes in Electrical Engineering. pp. 15-24. Springer, New York (2020)

20. Karuppasamy, P., Abudhahir, A., Prabhakaran, M., Thirunavukkarasu, S., Rao, B.P.C., Jayakumar, T.: Modelbased optimization of MFL testing of ferromagnetic steam generator tubes. J. Nondestr. Eval. 35, 1-9 (2016). https://doi.org/ 10.1007/s10921-015-0320-x

21. Atherton, D.L.: Stress-shadow magnetic inspection technique for far-side anomalies in steel pipe. NDT Int. 16, 145-149 (1983). https://doi.org/10.1016/0308-9126(83)90037-8

22. Vértesy, G., Gasparics, A., Tomáš, I.: Inspection of local wall thinning by different magnetic methods. J. Nondestr. Eval. 37, 65 (2018). https://doi.org/10.1007/s10921-018-0515-Z

23. Tsukada, K., Yoshioka, M., Kawasaki, Y., Kiwa, T.: Detection of back-side pit on a ferrous plate by magnetic flux leakage method with analyzing magnetic field vector. NDT E Int. 43, 323-328 (2010). https://doi.org/10.1016/j.ndteint.2010.01.004

24. Sakai, K., Morita, K., Haga, Y., Kiwa, T., Inoue, K., Tsukada, K.: Automatic scanning system for back-side defect of steel structure using magnetic flux leakage method. IEEE Trans. Magn. (2015). https://doi.org/10.1109/TMAG.2015.2453211

25. Kasai, N., Fujiwara, Y., Sekine, K., Sakamoto, T.: Evaluation of back-side flaws of the bottom plates of an oil-storage tank by the RFECT. NDT E Int. 41, 525-529 (2008). https://doi.org/10.1016/ j.ndteint.2008.05.002

26. Kasai, N., Maeda, T., Tamura, K., Kitsukawa, S., Sekine, K.: Application of risk curve for statistical analysis of backside corrosion in the bottom floors of oil storage tanks. Int. J. Press. Vessel. Pip. 141, 19-25 (2016). https://doi.org/10.1016/j.ijpvp.2016.03.014

27. Deng, Z., Sun, Y., Kang, Y., Song, K., Wang, R.: A permeabilitymeasuring magnetic flux leakage method for inner surface crack in thick-walled steel pipe. J. Nondestr. Eval. 36, 1-14 (2017). https:// doi.org/10.1007/s10921-017-0447-z

28. Zhang, Y., Sekine, K., Watanabe, S.: Magnetic leakage field due to sub-surface defects in ferromagnetic specimens. NDT E Int. 28, 67-71 (1995). https://doi.org/10.1016/0963-8695(94)00004-4

29. Wu, J., Wu, W., Li, E., Kang, Y.: Magnetic Flux Leakage Course of Inner Defects and Its Detectable Depth. Chin. J. Mech. Eng. 2021(341), 1-11 (2021). https://doi.org/10.1186/S10033021-00579-Y

30. Packer, S.A.H., Pearson, N.R., Priewald, R.H.: Methods and apparatus for the inspection of plates and pipe walls, https://worldwide. espacenet.com/patent/search/family/044067502/publication/ EP2506003A1?q=EP2506003A1 (2012)

31. Cheng, W.: Magnetic flux leakage testing of reverse side wallthinning by using very low strength magnetization. J. Nondestr. Eval. 35, 1-9 (2016). https://doi.org/10.1007/s10921-016-0347-7

32. Wang, Z.D., Yao, K., Deng, B., Ding, K.Q.: Theoretical studies of metal magnetic memory technique on magnetic flux leakage signals. NDT E Int. 43, 354-359 (2010). https://doi.org/10.1016/j. ndteint.2009.12.006

33. Babbar, V., Clapham, L.: Residual magnetic flux leakage: a possible tool for studying pipeline defects. J. Nondestr. Eval. 22, 117-125 (2003). https://doi.org/10.1023/B:JONE.0000022031.16580.5a

Publisher's Note Springer Nature remains neutral with regard to jurisdictional claims in published maps and institutional affiliations. 